\title{
CURVATURE ANALYSIS OF PATTERN TRANSFORMATION MANIFOLDS
}

\author{
Elif Vural and Pascal Frossard \\ Ecole Polytechnique Fédérale de Lausanne (EPFL) \\ Signal Processing Laboratory - LTS4 \\ CH-1015 Lausanne, Switzerland
}

\begin{abstract}
Transformation manifolds are quite attractive for image analysis applications that require transformation invariance properties. The geometric structure of a transformation manifold has a profound influence on the design of processing algorithm, and the curvature is a major parameter in the characterization of the manifold geometry. We propose here a procedure for the computation of an upper bound for the maximum principal curvature of a pattern transformation manifold. We provide an analytical formulation of the curvature bound and show that the numerical computation of this bound is mostly dependent on the rotation parameters. Experimental results indicate that the curvature bound of the manifold has considerable dependence on the spatial complexity and smoothness of the generating pattern. Moreover, experiments with discretization of manifolds suggest that the curvature of the manifold is likely to affect the accuracy of compact representation and sampling algorithms.
\end{abstract}

Index Terms - Pattern transformation manifolds, Riemannian manifolds, principal curvature, transformation-invariance

\section{INTRODUCTION}

Modeling sets of signals via manifolds not only provides concise representations for data, but also helps to understand their structure. A family of signals definable by a mapping from a small set of parameters to the high-dimensional signal space constitutes a manifold. Among several manifold models for visual signals, transformation manifolds are of specific interest. They define the region of space formed by all transformations of a reference visual pattern and hence permit transformation-invariant analysis of images exposed to geometric transformations.

A critical factor affecting the treatment of a manifold-modeled signal is the geometric structure of the manifold. Local properties such as differentiability and smoothness typically have significant influence on the performance of algorithms that focus on sampling, manifold distance computation or image analysis. For instance, in [1] Wakin et al. show that the articulation manifolds generated by images with sharp edges are nowhere differentiable, therefore the registration of such signals requires the utilization of multiscale methods.

In this work, we focus on the curvature analysis of pattern transformation manifolds defined by the in-plane rotation, translation and isotropic scaling of two-dimensional patterns. Being a measure of smoothness, the curvature of a manifold provides crucial information for its characterization. In [2] a method is proposed for the

This work has been partly funded by the Swiss National Science Fundation under grant number 200021_120060. numerical estimation of the principal curvature at a specified manifold point and normal direction. On the other hand, we examine the maximum value of the principal curvature over the whole manifold, and show that an overall upper bound for the principal curvature can be computed with a one-dimensional search over the rotation parameter. We illustrate the evolution of the proposed curvature bound for characterizing manifold smoothness by experimentation on patterns of different spatial complexity. In addition, results of image registration experiments on the discretization of pattern transformation manifolds suggest that such a curvature bound may provide information about the compactness or accuracy of the discrete manifold representation.

\section{ANALYTICAL FORMULATION OF THE CURVATURE BOUND}

Given a visual pattern $p$, we examine the curvature of the pattern transformation manifold $\mathcal{M}$ generated by applying $p$ geometric transformations composed of rotation, translation and isotropic scaling. Let $p=p(\tilde{x}, \tilde{y}) \in L^{2}\left(\mathbb{R}^{2}\right)$ be a differentiable function representing a visual pattern. We define our transformation manifold $\mathcal{M} \subset L^{2}\left(\mathbb{R}^{2}\right)$ on the compact domain $\Lambda \subset \mathbb{R}^{4}$ of parameter vectors,

$$
\begin{aligned}
\Lambda=\left\{\lambda ; \lambda=\left(\theta, t_{x}, t_{y}, s\right), \theta\right. & \in\left[\theta_{l}, \theta_{u}\right], t_{x} \in\left[t_{x l}, t_{x u}\right], \\
t_{y} & \left.\in\left[t_{y l}, t_{y u}\right], s \in\left[s_{l}, s_{u}\right]\right\},
\end{aligned}
$$

where $\theta$ denotes the rotation, $t_{x}$ and $t_{y}$ describe the translation in the horizontal and vertical directions, and $s$ represents the isotropic scaling. Then $\mathcal{M}$ is given by

$$
\mathcal{M}=\left\{g_{\lambda}: g_{\lambda}=U_{\lambda}(p), \lambda=\left(\theta, t_{x}, t_{y}, s\right) \in \Lambda\right\} \subset L^{2}\left(\mathbb{R}^{2}\right),
$$

where $g_{\lambda}$ is the pattern obtained by applying the transformation $U_{\lambda}$ specified by $\lambda$ to $p$. The relation between $g_{\lambda}$ and $p$ can be formulated by the change of variables $g_{\lambda}(x, y)=p(\tilde{x}, \tilde{y})$, where

$$
\left[\begin{array}{l}
x \\
y
\end{array}\right]=\left[\begin{array}{cc}
\cos \theta & -\sin \theta \\
\sin \theta & \cos \theta
\end{array}\right]\left[\begin{array}{ll}
s & 0 \\
0 & s
\end{array}\right]\left[\begin{array}{l}
\tilde{x} \\
\tilde{y}
\end{array}\right]+\left[\begin{array}{l}
t_{x} \\
t_{y}
\end{array}\right] .
$$

Since $p(\tilde{x}, \tilde{y}) \in L^{2}\left(\mathbb{R}^{2}\right)$ is differentiable with respect to $\tilde{x}$ and $\tilde{y}$ and the mapping between the variables $(x, y)$ and $(\tilde{x}, \tilde{y})$ is also differentiable, it follows that $\mathcal{M}$ is a differentiable manifold and a Riemannian manifold in particular. Now, we base our analysis on the following definition of curvature on a Riemannian manifold [3], [4]: Let $g_{\lambda} \in \mathcal{M}$ be a manifold point and $T_{g_{\lambda}}$ denote the tangent space at $g_{\lambda}$. There exists a symmetric bilinear form $B: T_{g_{\lambda}} \times T_{g_{\lambda}} \rightarrow T_{g_{\lambda}}^{\perp}$ that maps every two tangent vectors to a normal vector at $g_{\lambda}$. For any unit normal vector $\eta \in T_{g_{\lambda}}^{\perp}$, one can define a linear self-adjoint operator $L_{\eta}: T_{g_{\lambda}} \rightarrow \mathbb{R}$ such that $B_{\eta}(u, v)=\langle\eta, B(u, v)\rangle=$ 
$\left\langle u, L_{\eta} v\right\rangle ; u, v \in T_{g_{\lambda}}$. For notational convenience, $u$ and $\eta$ will denote unit norm vectors throughout the analysis. The maximum principal curvature $\kappa_{\eta}$ at $g_{\lambda}$ in the normal direction $\eta$ is the norm of the second fundamental form, i.e., the largest eigenvalue of $L_{\eta}$, given by

$$
\kappa_{\eta}\left(g_{\lambda}\right)=\sup _{u \in T_{g_{\lambda}},\|u\|=1}\left\langle u, L_{\eta} u\right\rangle .
$$

In order to achieve an overall curvature characterization of the manifold, we seek an upper bound for the supremum value $\mathcal{K}$ of the principal curvature,

$$
\mathcal{K}=\sup _{g_{\lambda} \in \mathcal{M}, \eta \in T_{g_{\lambda}}^{\perp}} \kappa_{\eta}\left(g_{\lambda}\right)=\sup _{\substack{g_{\lambda} \in \mathcal{M}, u \in T_{g_{\lambda}} \\ \eta \in T_{g_{\lambda}}^{\perp}}}\langle\eta, B(u, u)\rangle .
$$

Now, let $g_{\gamma_{u}(t)} \in \mathcal{M}$ be the geodesic curve on $\mathcal{M}$ starting at $g_{\lambda}$ with unit velocity $u \in T_{g_{\lambda}}$ at $g_{\lambda}$, such that $\gamma_{u}:[0, \delta] \rightarrow \Lambda$, $\gamma_{u}(0)=\lambda$. We assume that $g_{\gamma_{u}(t)}$ is arc-length parameterized, i.e. $\left\|\partial_{t} g_{\gamma_{u}(t)}\right\|=\left\|\sum_{i} \partial_{i} g_{\gamma_{u}(t)} \partial_{t} \gamma_{u}^{i}\right\|=1$ for every $t \in[0, \delta]$, where $\partial_{i}($.$) denotes partial differentiation with respect to the i^{t h}$ local coordinate and the notation $\|$.$\| denotes the L^{2}$-norm throughout the analysis. In the computation of an upper bound for curvature, we will make use of the following identity:

\section{Proposition 1.}

$$
\mathcal{K}=\sup _{g_{\lambda} \in \mathcal{M}, \eta \in T_{g_{\lambda}}^{\perp}} \kappa_{\eta}\left(g_{\lambda}\right)=\sup _{g_{\lambda} \in \mathcal{M}, u \in T_{g_{\lambda}}}\left\|\left.\partial_{t t} g_{\gamma_{u}(t)}\right|_{t=0}\right\| .
$$

Proof. Following the standard definition [3] of the symmetric bilinear form $B(u, v)$, we have

$$
\begin{aligned}
B(u, u) & =\left.B\left(\partial_{t} g_{\gamma_{u}(t)}, \partial_{t} g_{\gamma_{u}(t)}\right)\right|_{t=0} \\
& =\left.\left(\bar{\nabla}_{\partial_{t} g_{\gamma_{u}(t)}} \partial_{t} g_{\gamma_{u}(t)}-\nabla_{\partial_{t} g_{\gamma_{u}(t)}} \partial_{t} g_{\gamma_{u}(t)}\right)\right|_{t=0} \\
& =\left.\left(\bar{\nabla}_{\partial_{t} g_{\gamma_{u}(t)}} \partial_{t} g_{\gamma_{u}(t)}\right)\right|_{t=0}=\left.\partial_{t t} g_{\gamma_{u}(t)}\right|_{t=0} \in T_{g_{\lambda}}^{\perp},
\end{aligned}
$$

where $\nabla$ and $\bar{\nabla}$ denote the Riemannian connections on $\mathcal{M}$ and $L^{2}\left(\mathbb{R}^{2}\right)$ respectively. The third equality follows from the fact that $g_{\gamma_{u}(t)}$ is a geodesic curve. Then, for any unit normal vector $\eta \in T_{g_{\lambda}}^{\perp}$,

$$
\langle\eta, B(u, u)\rangle \leq\|\eta\|\left\|\left.\partial_{t t} g_{\gamma_{u}(t)}\right|_{t=0}\right\|=\left\|\left.\partial_{t t} g_{\gamma_{u}(t)}\right|_{t=0}\right\|,
$$

where the equality in (5) is attained for the choice of unit normal as $\eta=\partial_{t t} g_{\gamma_{u}(t)} /\left.\left\|\partial_{t t} g_{\gamma_{u}(t)}\right\|\right|_{t=0}$.

Following the statement of Prop. 1, we would like to find a bound $A$ such that $\mathcal{K}=\sup _{\lambda \in \Lambda, u \in T_{g_{\lambda}}}\left\|\left.\partial_{t t} g_{\gamma_{u}(t)}\right|_{t=0}\right\| \leq A$. In [5] Jacques et al. show that ${ }^{1}$ at any point $g_{\lambda} \in \mathcal{M}$

$$
\sup _{u \in T_{g_{\lambda}}}\left\|\left.\partial_{t t} g_{\gamma_{u}(t)}\right|_{t=0}\right\|^{2} \leq\left\langle\partial_{i j} g_{\lambda}, \partial_{k l} g_{\lambda}\right\rangle \mathcal{G}^{i k} \mathcal{G}^{j l}
$$

using the Einstein summation notation, where $\partial_{i} g_{\lambda}=\partial g_{\lambda} / \partial \lambda_{i}$ and $\partial_{i j} g_{\lambda}=\partial^{2} g_{\lambda} / \partial \lambda_{i} \partial \lambda_{j}$ are the first and second order partial derivatives at $g_{\lambda}$ with respect to the local coordinates in the parameter domain $\Lambda ; \mathcal{G}_{i j}$ is the metric tensor on $\mathcal{M}$ defined by $\mathcal{G}_{i j}=$ $\left\langle\partial_{i} g_{\lambda}, \partial_{j} g_{\lambda}\right\rangle$; and $\left[\mathcal{G}^{i j}\right]$ is the inverse of the matrix $\left[\mathcal{G}_{i j}\right]$. Since our

\footnotetext{
${ }^{1}$ Although the proof in [5] is intended for dictionary atoms of unit norm, the given bound is derived based on geodesic properties and is valid for Riemannian manifolds in general.
}

pattern transformation manifold has intrinsic dimension 4, (6) implies that

$\mathcal{K}=\sup _{\lambda \in \Lambda, u \in T_{g_{\lambda}}}\left\|\left.\partial_{t t} g_{\gamma_{u}(t)}\right|_{t=0}\right\| \leq 4^{2} \sup _{\lambda \in \Lambda, i, j}\left\|\partial_{i j} g_{\lambda}\right\| \sup _{\lambda \in \Lambda, i, j}\left|\mathcal{G}^{i j}\right|$.

Now, assuming that the reference pattern $p(\tilde{x}, \tilde{y}) \in L^{2}\left(\mathbb{R}^{2}\right)$ is nonzero only within the region $\tilde{I}=[-W / 2, W / 2] \times[-H / 2, H / 2]$, we present an upper bound $S_{1}$ such that $\sup _{\lambda \in \Lambda, i, j}\left\|\partial_{i j} g_{\lambda}\right\| \leq S_{1}$ in Sec. 2.1, and we give in Sec. 2.2 the formulation of an upper bound $\sup _{\lambda \in \Lambda, i, j}\left|\mathcal{G}^{i j}\right| \leq S_{2}$. Using (7), $S_{1}$ and $S_{2}$ together provide an upper bound for the maximum principal curvature as $\mathcal{K} \leq 16 S_{1} S_{2}$.

\subsection{Upper bound for $\sup _{\lambda \in \Lambda, i, j}\left\|\partial_{i j} g_{\lambda}\right\|$}

Observe that from (2),

$$
\begin{aligned}
g_{\lambda}(x, y)=p(\tilde{x}, \tilde{y}) & =p\left(\frac{1}{s} \cos \theta\left(x-t_{x}\right)+\frac{1}{s} \sin \theta\left(y-t_{y}\right),\right. \\
& \left.-\frac{1}{s} \sin \theta\left(x-t_{x}\right)+\frac{1}{s} \cos \theta\left(y-t_{y}\right)\right),
\end{aligned}
$$

where $\tilde{x}$ and $\tilde{y}$ are written in terms of $x$ and $y$ and the transformation parameters. This gives

$$
\partial_{i} g_{\lambda}(x, y)=\partial_{\tilde{x}} p \partial_{i} \tilde{x}+\partial_{\tilde{y}} p \partial_{i} \tilde{y}
$$

$$
\begin{aligned}
\partial_{i j} g_{\lambda}(x, y) & =\partial_{\tilde{x} \tilde{x}} p \partial_{i} \tilde{x} \partial_{j} \tilde{x}+\partial_{\tilde{x} \tilde{y}} p \partial_{j} \tilde{x} \partial_{i} \tilde{y}+\partial_{\tilde{x} \tilde{y}} p \partial_{i} \tilde{x} \partial_{j} \tilde{y} \\
& +\partial_{\tilde{y} \tilde{y}} p \partial_{i} \tilde{y} \partial_{j} \tilde{y}+\partial_{\tilde{x}} p \partial_{i j} \tilde{x}+\partial_{\tilde{y}} p \partial_{i j} \tilde{y}
\end{aligned}
$$

From (2) it follows that

$$
\partial_{\theta} \tilde{x}=-\frac{1}{s} \sin \theta\left(x-t_{x}\right)+\frac{1}{s} \cos \theta\left(y-t_{y}\right)=\tilde{y},
$$

and when similar expressions for the remaining $\partial_{i} \tilde{x}$ and $\partial_{i} \tilde{y}$ are obtained, it is seen that all partial derivatives can be written in terms of $\tilde{x}, \tilde{y}, s$ and $\theta$; such that they are all independent of the translation parameters $t_{x}$ and $t_{y}$. Now let the support region $\tilde{I}=[-W / 2, W / 2] \times$ $[-H / 2, H / 2]$ of $p(\tilde{x}, \tilde{y})$ be mapped to the region $I$ by the transformation $p \rightarrow g_{\lambda}$. Then,

$$
\begin{aligned}
\left\|\partial_{i j} g_{\lambda}\right\|^{2} & =\int_{I}\left(\partial_{i j} g_{\lambda}(x, y)\right)^{2} d x d y \\
& =s^{2} \int_{\tilde{I}}\left(\partial_{i j} g_{\lambda}(x(\tilde{x}, \tilde{y}), y(\tilde{x}, \tilde{y}))\right)^{2} d \tilde{x} d \tilde{y},
\end{aligned}
$$

where the Jacobian of the transformation is related to the scale parameter as $\left|\frac{\partial(x, y)}{\partial(\tilde{x}, \tilde{y})}\right|=s^{2}$. Then from (12) it is easy to show that

$$
\sup _{\lambda \in \Lambda, i, j}\left\|\partial_{i j} g_{\lambda}\right\| \leq \sqrt{W H} s_{u} \sup _{\lambda \in \Lambda, i, j, x, y}\left|\partial_{i j} g_{\lambda}(x, y)\right| .
$$

Examination of the partial derivatives of $\tilde{x}$ and $\tilde{y}$ with respect to the transformation parameters as in (11) indicates that $\left|\partial_{i} \tilde{x}\right|,\left|\partial_{i} \tilde{y}\right| \leq$ $\max (W, H) \max \left(s_{l}^{-1}, 1\right)$, and $\left|\partial_{i j} \tilde{x}\right|,\left|\partial_{i j} \tilde{y}\right| \leq 2 \max (W, \bar{H})$ $\max \left(s_{l}^{-2}, 1\right)$ for every $i, j$. From equation (10)

$$
\left|\partial_{i j} g_{\lambda}(x, y)\right| \leq 4 \sup _{z, w}\left|\partial_{z w} p\right| \sup _{z, i}\left|\partial_{i} z\right|^{2}+2 \sup _{z}\left|\partial_{z} p\right| \sup _{z, i, j}\left|\partial_{i j} z\right|,
$$

where $z, w \in\{\tilde{x}, \tilde{y}\}$. Combining these with (13) gives

$$
\begin{gathered}
\sup _{\lambda \in \Lambda, i, j}\left\|\partial_{i j} g_{\lambda}\right\| \leq S_{1}=\sqrt{W H} s_{u}\left(4 \sup _{z, w}\left|\partial_{z w} p\right| \max \left(W^{2}, H^{2}\right)\right. \\
\left.\max \left(s_{l}^{-2}, 1\right)+4 \sup _{z}\left|\partial_{z} p\right| \max (W, H) \max \left(s_{l}^{-2}, 1\right)\right) .
\end{gathered}
$$




\subsection{Upper bound for $\sup _{\lambda \in \Lambda, i, j}\left|\mathcal{G}^{i j}\right|$}

We would like to find an upper bound for the norm of the entries of the matrix $\left[\mathcal{G}^{i j}\right]=\left[\mathcal{G}_{i j}\right]^{-1}$. Both $\left[\mathcal{G}_{i j}\right]$ and $\left[\mathcal{G}^{i j}\right]$ are symmetric positive definite matrices, their diagonal entries are positive, and the eigenvalues of the two matrices are the inverses of each other. Therefore, the magnitudes of the entries of $\left[\mathcal{G}^{i j}\right]$ are bounded by

$$
\left|\mathcal{G}^{i j}\right| \leq \operatorname{tr}\left(\left[\mathcal{G}^{i j}\right]\right)=\sum_{k=1}^{4} \lambda_{k}^{-1}\left(\left[\mathcal{G}_{i j}\right]\right) \leq 4 \lambda_{\text {min }}^{-1}\left(\left[\mathcal{G}_{i j}\right]\right),
$$

where $\lambda_{k}($.$) denotes the k^{t h}$ eigenvalue of a matrix, and $\lambda_{\min }($. denotes the smallest eigenvalue of the matrix. It is possible to show that (see Sec. 3.2) the entries of the matrix $\left[\mathcal{G}_{i j}\right]$ involve only $s$ and $\theta$, and not the translation parameters, therefore can be represented as $\left[\mathcal{G}_{i j}(\theta, s)\right]$. Moreover, the entries of $\left[\mathcal{G}^{i j}\right]$ attain their largest magnitudes when the scale $s$ is at its smallest value $s_{l}$. Combining this observation with (14) gives the following result:

$$
\sup _{\lambda \in \Lambda, i, j}\left|\mathcal{G}^{i j}\right| \leq S_{2}=\frac{4}{\inf _{\theta}\left(\lambda_{\min }\left(\left[\mathcal{G}_{i j}\left(\theta, s_{l}\right)\right]\right)\right)} .
$$

\section{NUMERICAL COMPUTATION OF THE CURVATURE BOUND}

Although the analytical bounds derived in Sec. 2 establish the representation of a curvature bound in terms of the reference pattern and transformation parameters, our derivation of the terms $S_{1}$ and $S_{2}$ involves extremal values of the image and transformation parameters, which may consequently cause the bound to be loose in some cases. However, we now discuss how a sharper curvature bound can be computed numerically. From (6) one can obtain

$$
\mathcal{K}^{2} \leq \sum_{i, j, k, l=1}^{4} \sup _{\lambda \in \Lambda}\left\|\partial_{i j} g_{\lambda}\right\| \sup _{\lambda \in \Lambda}|| \partial_{k l} g_{\lambda}|| \sup _{\lambda \in \Lambda}\left|\mathcal{G}^{i k}\right| \sup _{\lambda \in \Lambda}\left|\mathcal{G}^{j l}\right|
$$

Based on this formulation, the numerical calculation of a curvature bound is possible by computing $\sup _{\lambda \in \Lambda} \| \partial_{i j} g_{\lambda}||$ and $\sup _{\lambda \in \Lambda}\left|\mathcal{G}^{i j}\right|$ for all combinations of $(i, j)$, and then calculating the summation in (16). In particular we show that the bound is independent of the translation parameters. It further has a simple dependency on the scale parameter, which permits to cast the bound computation as a one-dimensional search over the rotation parameter. We describe now the numerical computation of $\sup _{\lambda \in \Lambda}\left\|\partial_{i j} g_{\lambda}\right\|$ and $\sup _{\lambda \in \Lambda}\left|\mathcal{G}^{i j}\right|$ for all possible pairs $(i, j)$.

\subsection{Computation of $\sup _{\lambda \in \Lambda}\left\|\partial_{i j} g_{\lambda}\right\|$}

Combining (10), (12) and the fact that the partial derivatives $\partial_{i} \tilde{x}$, $\partial_{i} \tilde{y}, \partial_{i j} \tilde{x}, \partial_{i j} \tilde{y}$ are independent of the translation parameters $t_{x}, t_{y}$; $\sup _{\lambda \in \Lambda}\left\|\partial_{i j} g_{\lambda}\right\|$ can already be estimated by a two-dimensional search over the rotation and scale parameters $\theta, s$. Now we show that one can also dispense with the search over the scale $s$.

In the first case " $\lambda_{i}=\lambda_{j}=\theta$ ", all partial derivatives $\partial_{i} \tilde{x}, \partial_{j} \tilde{x}$, $\partial_{i} \tilde{y}, \partial_{j} \tilde{y}, \partial_{i j} \tilde{x}, \partial_{i j} \tilde{y}$ are independent of $s$, hence, the integration in (12) is of the form $\left\|\partial_{i j} g_{\lambda}\right\|^{2}=s^{2} F(\theta)$. Therefore $\left\|\partial_{i j} g_{\lambda}\right\|$ reaches its supremum at $s=s_{u}$. Similarly, if we examine the second case " $\lambda_{i}=\theta, \lambda_{j} \neq \theta$ ", then $\partial_{i} \tilde{x}, \partial_{i} \tilde{y}$ are independent of $s$, i.e. in the form $f(\theta, \tilde{x}, \tilde{y})$; and $\partial_{j} \tilde{x}, \partial_{j} \tilde{y}, \partial_{i j} \tilde{x}, \partial_{i j} \tilde{y}$ are of the form $f(\theta, \tilde{x}, \tilde{y}) s^{-1}$. Therefore, the integration in (12) has the form $\left\|\partial_{i j} g_{\lambda}\right\|^{2}=s^{2} \int_{\tilde{I}} g(\theta, \tilde{x}, \tilde{y})^{2} s^{-2} d \tilde{x} d \tilde{y}=F(\theta)$, and is independent of the scale parameter. In a similar manner, one can show that $\left\|\partial_{i j} g_{\lambda}\right\|$ reaches its supremum at $s=s_{l}$ in the other cases.

\subsection{Computation of $\sup _{\lambda \in \Lambda}\left|\mathcal{G}^{i j}\right|$}

We now show that bounds for the magnitudes of the entries of $\left[\mathcal{G}^{i j}\right]$ can also be estimated by a one dimensional search over the rotation parameter. From (9) one can construct the metric tensor as

$$
\mathcal{G}_{i j}=s^{2} \int_{\tilde{I}}\left(\partial_{\tilde{x}} p \partial_{i} \tilde{x}+\partial_{\tilde{y}} p \partial_{i} \tilde{y}\right)\left(\partial_{\tilde{x}} p \partial_{j} \tilde{x}+\partial_{\tilde{y}} p \partial_{j} \tilde{y}\right) d \tilde{x} d \tilde{y}
$$

for instance, $\mathcal{G}_{\theta \theta}=s^{2} \int_{\tilde{I}}\left(\partial_{\tilde{x}} p \tilde{y}-\partial_{\tilde{y}} p \tilde{x}\right)^{2} d \tilde{x} d \tilde{y}$. Since all entries of $\left[\mathcal{G}_{i j}\right]$ are independent of the translation parameters $t_{x}, t_{y}$, one can use the notation $\left[\mathcal{G}_{i j}(s, \theta)\right]$. Moreover, all entries can be decomposed in the form $\mathcal{G}_{i j}(s, \theta)=f_{i j}(s) F_{i j}(\theta)$, where $f_{11}=s^{2}$; $f_{1 j}=f_{j 1}=s$ if $j \neq 1$; and $f_{i j}=1$ if $i, j \neq 1$. If we denote the matrix with entries $F_{i j}(\theta)$ by $\left[F_{i j}(\theta)\right]$, then from the linearity of the determinant with respect to the rows and columns, we have $\operatorname{det}\left(\left[\mathcal{G}_{i j}(s, \theta)\right]\right)=s^{2} \operatorname{det}\left(\left[F_{i j}(\theta)\right]\right)$. Now let us denote the $(i, j)^{t h}$ minor of $\left[\mathcal{G}_{i j}(s, \theta)\right]$ by $M_{i j}^{\mathcal{G}}(s, \theta)$, and the $(i, j)^{t h}$ minor of $\left[F_{i j}(\theta)\right]$ by $M_{i j}^{F}(\theta)$. Then, one can write $M_{i j}^{\mathcal{G}}(s, \theta)=m_{i j}(s) M^{F}{ }_{i j}(\theta)$, where $m_{i j}(s) \in\left\{1, s, s^{2}\right\} \forall i, j$. The magnitude of the entries of the matrix $\left[\mathcal{G}^{i j}(s, \theta)\right]$ are given by

$$
\begin{aligned}
\left|\mathcal{G}^{i j}(s, \theta)\right| & =\frac{\left|(-1)^{i+j} \operatorname{det}\left(\left[M_{j i}^{\mathcal{G}}(s, \theta)\right]\right)\right|}{\left|\operatorname{det}\left(\left[\mathcal{G}_{i j}(s, \theta)\right]\right)\right|}=\frac{\left|m_{j i}(s) M^{F}{ }_{j i}(\theta)\right|}{\mid s^{2} \operatorname{det}\left(\left[F_{i j}(\theta)\right] \mid\right.} \\
& =p_{j i}(s) \frac{\left|M^{F}{ }_{j i}(\theta)\right|}{\mid \operatorname{det}\left(\left[F_{i j}(\theta)\right] \mid\right.}=p_{j i}(s)\left|F_{i j}^{-1}(\theta)\right|
\end{aligned}
$$

where $p_{i j}(s)=m_{i j}(s) / s^{2} \in\left\{1, s^{-1}, s^{-2}\right\} \forall i, j$; and $\left[F_{i j}^{-1}(\theta)\right]$ is the inverse of the matrix $\left[F_{i j}(\theta)\right]$. It is clear that all entries of $\left[\mathcal{G}^{i j}(s, \theta)\right]$ attain their largest magnitude at $s=s_{l}$. Therefore it is possible to find upper bounds for the entries with a one-dimensional search over the rotation parameter $\theta$. This result and the conclusions of Sec. 3.1 together provide a solution for the computation a global bound for the principal curvature through (16).

\section{EXPERIMENTAL RESULTS}

In this section, we experimentally examine the curvature bounds of transformation manifolds of patterns with varying characteristics. The experiments are conducted on the transformation manifolds of patterns derived from the four images shown in Fig. 1. In all experiments, two different curvature measures for the examined transformation manifold are calculated: Firstly, the computational procedure explained in Sec. 3 is employed to find a global upper bound for the principal curvature that is valid over the whole manifold. Then, the local bound (6) for the maximum principal curvature of a point is computed at sufficiently many randomly selected manifold points, and the largest of these local bounds is taken as a measure of manifold curvature (denoted as maximum local curvature bound in the results). These two curvature bounds are displayed together in all of the following experimental results.

First, the relation between the smoothness of a pattern and the curvature of its transformation manifold is examined. A sequence of patterns are created from the "leaves" image shown in Fig. 1(a) (downsampled to $57 \times 73$ pixels) by applying a $3 \times 3$ low-pass filter successive times, and curvature bounds for the transformation manifolds of each of these patterns are computed. All transformation manifolds are defined in the parameter domain $\theta \in[-\pi, \pi] ; t_{x}, t_{y} \in$ $[-30,30] ; s \in[0.5,1.5]$. In Fig. 2 the curvature bounds are displayed with respect to the number of filters applied to obtain the pattern. The results indicate that the local and global curvature bounds 


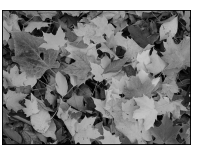

(a) Leaves

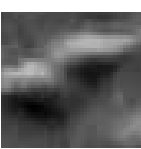

(b) Cell

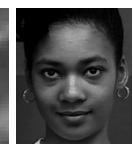

(c) Face

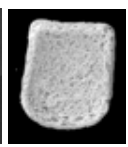

(d) Bread

Fig. 1. Patterns used in experimentation

change in a consistent manner and both decrease as the degree of filtering on the pattern is increased. This confirms that the smoothness of the manifold is directly related to the smoothness of the pattern.

Then, in a similar experiment, the influence of the complexity of the reference pattern over the manifold curvature is investigated. An expansion of the original image in terms of the atoms of a redundant dictionary is obtained using the Tree-Based Pursuit algorithm [6]. Then, following the order of the decomposition, the sequence of successively refined approximations of the original image is constructed from its projections on dictionary atoms, and curvature bounds are computed for the transformation manifolds of each of the patterns in the approximation sequence. The experiment is performed on the "cell" and "face" images shown in Fig. 1(b) and 1(c). Both images are downsampled to $32 \times 32$ pixels, and the transformation manifolds are defined over the parameter range $\theta \in[-\pi, \pi] ; t_{x}, t_{y} \in$ $[-20,20] ; s \in[0.5,1.5]$. The curvature bounds with respect to the number of atoms used in the approximation are plotted in Fig. 4(a) and 4(b), for the "cell" and "face" patterns respectively. As the number of atoms in the representation is increased, the image approximation includes more spatial detail. The figures show that the maximum local curvature bound follows the increase in the spatial complexity.

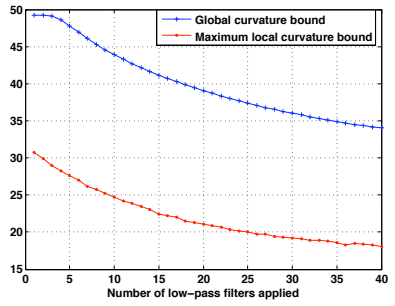

Fig. 2. Curvature bounds for the transformation manifolds of successively filtered patterns

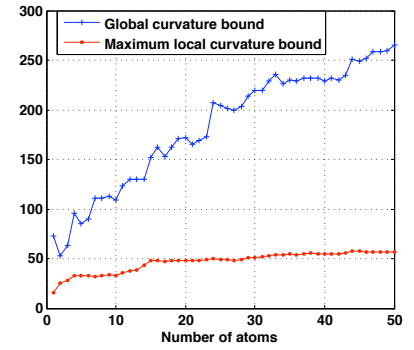

(a) Cell pattern

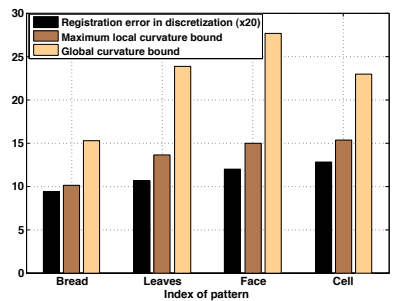

Fig. 3. Curvature bounds and the registration errors given by the manifold discretizations

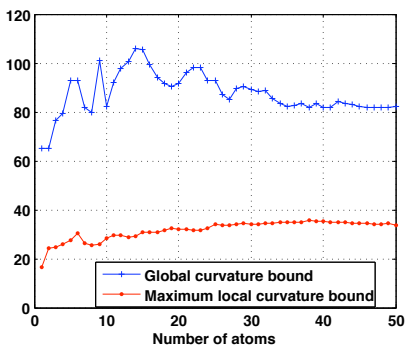

(b) Face pattern
Fig. 4. Curvature bounds for transformation manifolds of progressive approximations of two patterns in a dictionary

Finally, the influence of the curvature of a manifold on its amenability to algorithmic treatment is searched in a last experiment. The transformation manifolds of the four patterns displayed in Fig. 1 are sampled using the Registration-Efficient Manifold Discretization (REMD) algorithm proposed in [7]; and the registration error yielded by each discretization, which is regarded as the performance measure of the REMD algorithm, is compared to the curvature of the manifold. All images in Fig. 1 are downsampled to $32 \times 32$ pixels, normalized, and low-pass filtered to assure smoothness. The transformation manifolds of all images are constructed over the parameter domain $\theta \in[-\pi, \pi] ; t_{x}, t_{y} \in[-15,15] ; s=1$, and 16 samples from each manifold are selected with the REMD algorithm. The registration error for each discretization is computed by finding the average Euclidean distance between randomly selected manifold points and their corresponding nearest manifold samples. The two curvature bounds are plotted together with the registration error in Fig. 3. The curvature bounds are in general accordance with the registration performance of the discretization. This suggests that the curvature of a transformation manifold may affect the difficulty of processing or representing it.

\section{CONCLUSIONS}

We have proposed a procedure for computing an overall upper bound for the maximum principal curvature of the transformation manifold generated by the rotation, translation and isotropic scaling of a visual pattern. We show that the computation of such a global curvature bound is achievable through a one-dimensional search over the rotation parameter, although the manifold has an intrinsic dimension of four. We experimentally show that an increase in the spatial intricacy of the pattern in general leads to a rise in the manifold curvature. Moreover, the experimentation on the sampling of transformation manifolds suggests that the curvature bound of a manifold may bear information about its liability to treatment or the ease of representing it through a discretization.

\section{ACKNOWLEDGMENTS}

The authors would like to thank Dr. Effrosyni Kokiopoulou for her valuable comments on the improvement of the text.

\section{REFERENCES}

[1] M. B. Wakin, D. L. Donoho, H. Choi, and R. G. Baraniuk, "The multiscale structure of non-differentiable image manifolds," in Proc. Wavelets XI at SPIE Optics and Photonics, 2005.

[2] E. Kokiopoulou, D. Kressner, and P. Frossard, "Optimal image alignment with random projections of manifolds: algorithm and geometric analysis," Submitted to IEEE Transactions on Image Processing, draft available at http://www.sam.math.ethz.ch/reports/2009/41.

[3] M. Do Carmo, Riemannian Geometry, Birkhäuser, Boston, MA, USA, 1992.

[4] P. Niyogi, S. Smale, and S. Weinberger, "Finding the homology of submanifolds with high confidence from random samples," Discrete and Computational Geometry, vol. 39, no. 1, pp. 419-441, 2008.

[5] L. Jacques and C. De Vleeschouwer, "A geometrical study of matching pursuit parametrization," IEEE Transactions on Signal Processing, vol. 56, no. 7, pp. 2835-2848, July 2008.

[6] P. Jost, P. Vandergheynst, and P. Frossard, "Tree-Based Pursuit: Algorithm and Properties," IEEE Transactions on Signal Processing, vol. 54, no. 12, pp. 4685-4697, 2006.

[7] E. Vural and P. Frossard, "Distance-based discretization of parametric signal manifolds," in IEEE International Conference on Acoustics, Speech and Signal Processing, Dallas, TX, USA, 2010. 